\title{
Simulation of fluorescence radiation for Cherenkov observatories
}

\section{Morcuende*, J. Rosado, J. L. Contreras, F. Arqueros,}

IPARCOS Institute and EMFTEL Department, Universidad Complutense de Madrid, E-28040

Madrid, Spain

E-mail: dmorcuen@ucm.es

Several techniques inside Astroparticle Physics use Extensive Air Showers (EAS) as a proxy to study very energetic particles that reach Earth. EAS themselves are detected through different means, among them the measurement of Cherenkov or fluorescence radiation produced in them. A detailed simulation of EAS is mandatory to develop, test and use these techniques. In this respect the most popular simulation program is the CORSIKA code. This code includes the Cherenkov emission but not the fluorescence one. In a previous work, we introduced the fluorescence emission in CORSIKA and showed first quantitative results on the fluorescence contamination in Cherenkov telescopes, either imaging or non-imaging ones. In this paper, we report on progress made in this work and discuss possible future applications in several currently operating Cherenkov observatories as well as in the design of future innovative Astroparticle Physics observatories.

The New Era of Multi-Messenger Astrophysics - Asterics2019

25 - 29 March, 2019

Groningen, The Netherlands

\footnotetext{
* Speaker.
} 


\section{Motivation}

Monte Carlo (MC) simulations of cosmic-ray air showers are required to characterize the performance of Cherenkov telescopes, e.g., instrument response functions. CORSIKA [1] is the most used MC code for this purpose. It includes the detailed simulation of the Cherenkov light emission [2,3], whereas the air fluorescence radiation, which is within the same wavelength range but less efficient, has not been implemented yet in the official CORSIKA software. Indeed, the fluorescence component has so far been neglected in Cherenkov telescopes. However, the nextgeneration imaging atmospheric Cherenkov telescopes (IACTs) of the CTA Observatory [4] will achieve such a high sensitivity that the small contribution of fluorescence light may be significant in certain circumstances.

\section{Method}

We have implemented the fluorescence emission within the CORSIKA framework. The code is thoroughly described in [5]. It essentially generates fluorescence photons along the track of every simulated charged particle as well as at any point where a particle falls below the simulation cut-off. This allows for a simulation of the fluorescence emission with a level of detail as high as that of the Cherenkov emission. Unlike other codes used for the evaluation of the air fluorescence radiation from a simplified one-dimension EAS, this code takes into account the lateral profile of the air showers, which is relevant for the simulation of camera images registered by IACTs.

Information on fluorescence photons is written in the same way as Cherenkov photons, either in a standard CORSIKA output file or in the dedicated output format for IACTs. As an example, the obtained spatial distribution of fluorescence photons on ground for $10 \mathrm{TeV}$ gamma-ray vertical showers (averaged over 1000 events) is compared to that of Cherenkov photons in Fig. 1.
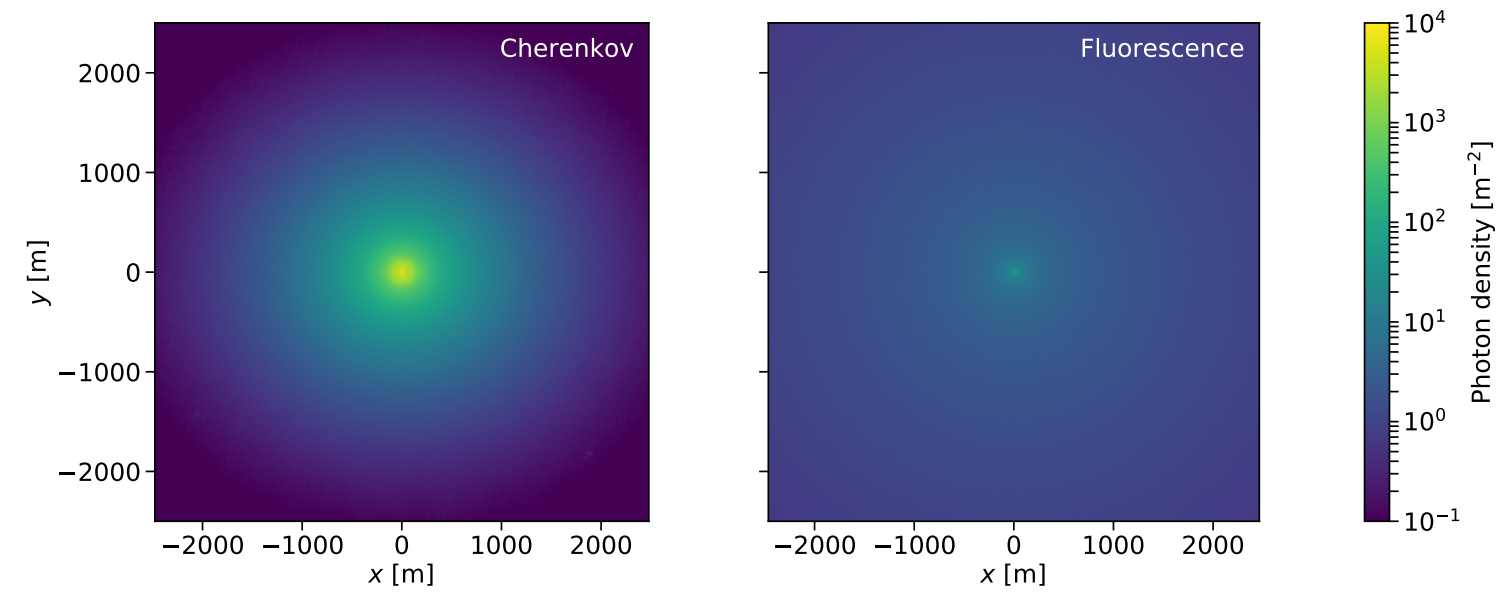

Figure 1: Spatial distributions of Cherenkov (left) and fluorescence (right) light on ground averaged over the simulation of $100010-\mathrm{TeV}$ gamma-ray vertical showers.

The simulation of Cherenkov emission consumes a large fraction of the CPU time used in CORSIKA. When including the fluorescence emission as well, the CPU time increases by a factor of 5-6 (e.g., from $240 \mathrm{~s}$ to $1400 \mathrm{~s}$ for 100 gamma showers of $1 \mathrm{TeV}$, for a $3300 \mathrm{MHz}$ CPU). 
The output file size also increases by around 6\% with respect to the simulation without producing fluorescence. Although it represents a substantial run-time increment, it is still feasible to perform dedicate MC productions thanks to the huge computational power available nowadays. Nonetheless, we are working on the optimization of the code with the aim to incorporate it into upcoming official CORSIKA v7 releases and, later on, in CORSIKA 8 [6]. Meanwhile, a modified CORSIKA code including fluorescence emission is available under request to the authors.

The MC tool sim_telarray [3] is extensively used along with CORSIKA (with the IACT output option) to perform end-to-end simulations for IACT systems. K. Bernlöhr has recently made this tool compatible with our code so as to include both Cherenkov and fluorescence photons in the telescope simulation. The user may also choose to use only one of the two light components. In this work, we present the first results using this new feature of sim_telarray.

\section{First results and on-going work}

We previously used our implementation of the fluorescence emission in CORSIKA to evaluate in a systematic way the relevance of the fluorescence radiation in Cherenkov telescopes [5]. This light contribution becomes important at large distances from the shower core impact point, because the Cherenkov light density decreases dramatically while that of the fluorescence light keeps rather constant due to its isotropic emission, as shown in Fig. 1. As an example, the red line in Fig. 2 represents the average ratio of the number of fluorescence photons to the total as a function of the core distance when restricting the photon arrival angles to the field of view of a typical IACT $\left(10^{\circ}\right.$ of angular diameter around the shower axis direction). The results were obtained for vertical

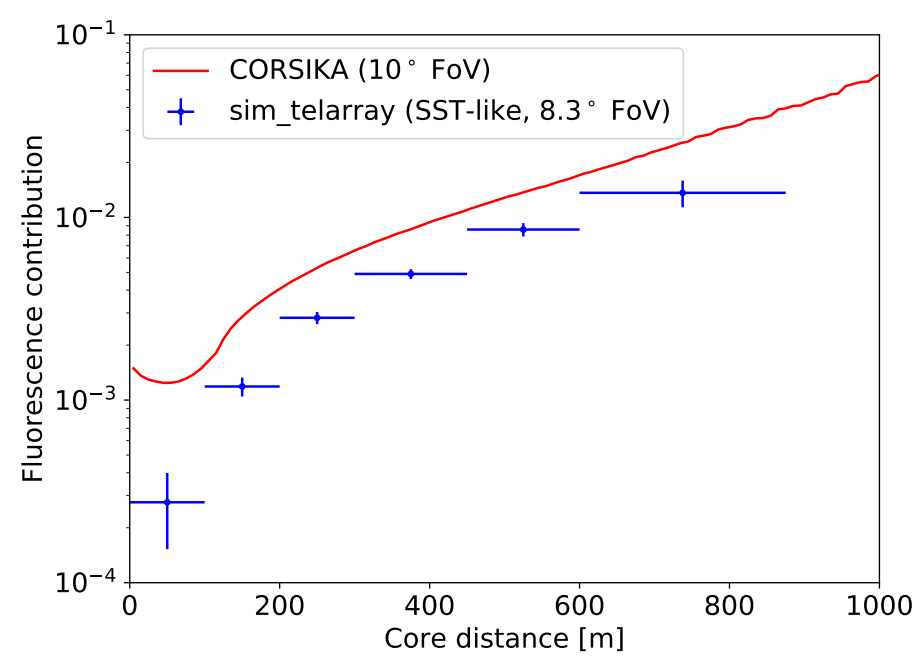

Figure 2: Contribution of fluorescence light to the total signal registered by SST-like CTA telescopes as a function of their distance to the shower impact point (in blue). The values were averaged over 6000 vertical showers initiated by $100-\mathrm{TeV}$ gamma rays. For comparison, the fluorescence contamination previously estimated in [5] solely from CORSIKA simulations (considering $10^{\circ} \mathrm{FoV}$ telescopes) is plotted in red. 
showers induced by $100-\mathrm{TeV}$ gamma rays. At about $1000 \mathrm{~m}$, the fluorescence contribution exceeds $5 \%$ of the total light.

These results are being cross-checked in a more realistic IACT scenario, by piping the CORSIKA output photons to sim_telarray. We simulate the layout, trigger and readout conditions of CTA. Preliminary results of the relative fluorescence contribution to the total amount of photoelectrons recorded by the telescopes is represented by blue crosses in Fig. 2. The values were averaged over 6000 vertical showers induced by $100-\mathrm{TeV}$ gamma rays and only the small-size telescopes (SST) were included in the simulation. Fluorescence contamination becomes $\gtrsim 1 \%$ at 700-800 m away from the shower core. This is consistent with the result obtained directly from the photon densities if taking into account the differences in the assumed field of view of the telescopes.

We have also used sim_telarray to simulate, for the first time, camera images from Cherenkov and fluorescence light separately. Examples are shown in Fig. 3 for a medium-size telescope (MST) and a SST. It can be appreciated that fluorescence light produces a similar-shape camera image, but with a smaller number of photo-electrons. In addition, the image from fluorescence light is broader and more noisy than the one from Cherenkov light. Although charged particles that are far from the shower axis do not induce Cherenkov light towards the direction of the telescope, they produce isotropic fluorescence light that can actually reach it.
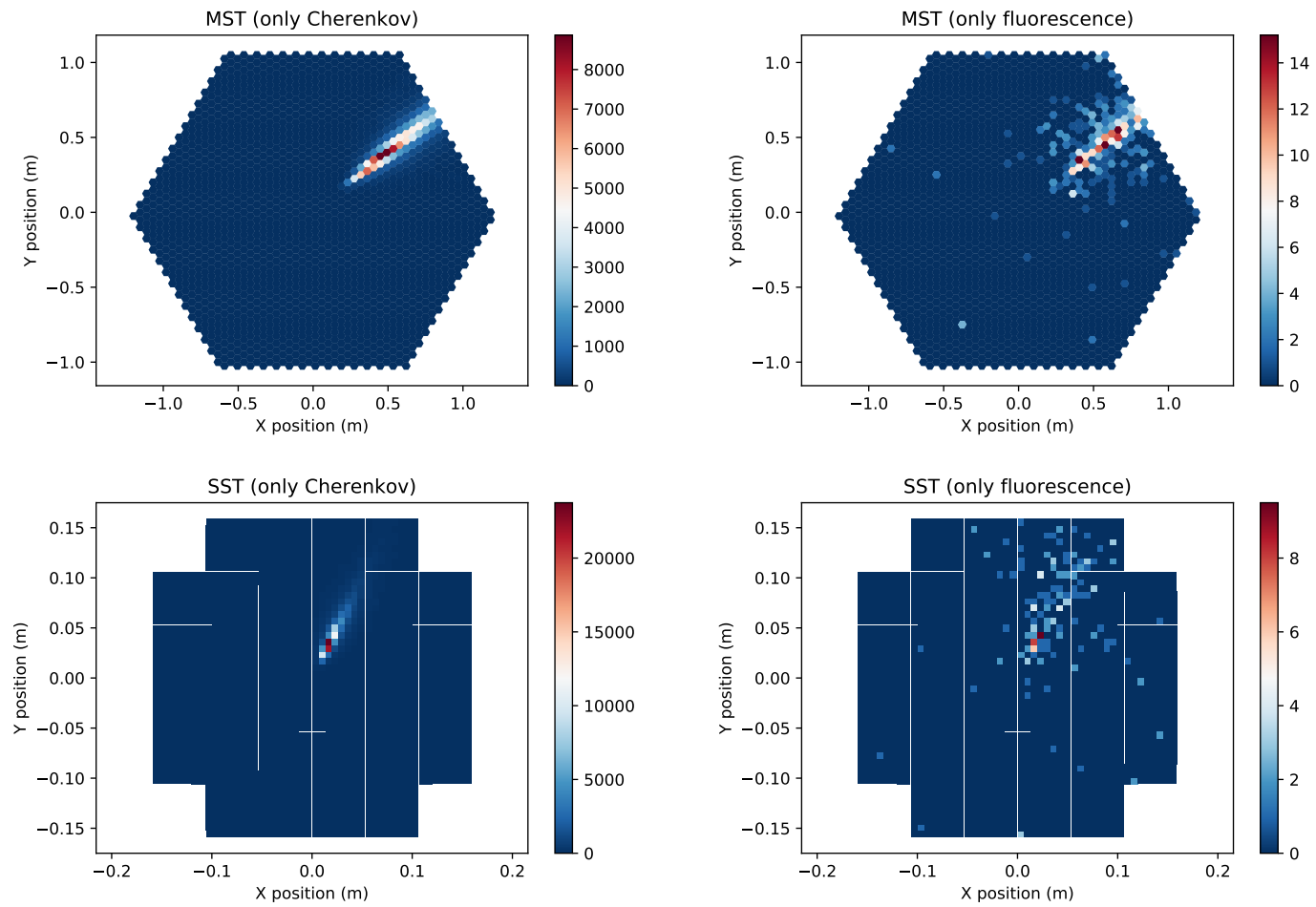

Figure 3: Simulated photo-electrons in the camera of a MST-like (top panels) and a SST-like (bottom panels) IACT from Cherenkov (left) and fluorescence (right) light produced in a 300-TeV gamma-ray induced vertical shower using the simulation tool sim_telarray. Both telescopes are located $\approx 200 \mathrm{~m}$ away from the shower impact point. These images do not contain night-sky background nor electronic noise.

We plan to extend these studies by analyzing the camera images with ctapipe, which is the 
prototype analysis pipeline being developed for CTA [9]. The first step is to assess the potential variations of Hillas parameters when including the fluorescence light in the simulations. Going further, we will better estimate the fluorescence contamination and evaluate the performance of Cherenkov telescopes when fluorescence light comes into play by making use of the whole analysis chain.

Moreover, we are exploring the possibility of using arrays of Cherenkov telescopes as fluorescence detectors. In this operation mode, fluorescence signal in IACTs is expected to be larger than that of Cherenkov. The telescopes would normally detect showers pointing to the gamma-ray sources, whereas they could simultaneously observe showers coming transversely from any other source. For this purpose, the trigger and readout systems must be adapted to the corresponding time window $(\sim 1-10 \mu \mathrm{s})$, as first discussed in [10]. We are carrying out a detailed MC study with sim_telarray to accurately determine these parameters. This operation mode of Cherenkov telescopes would potentially allow the registration of cosmic-ray showers for hadronic interaction studies as well as increasing the effective area for VHE gamma-ray astronomy.

\section{Acknowledgments}

This work has been supported by the Spanish MINECO (under the contracts FPA2015-69210C6-3-R and FPA2017-82729-C6-3-R), the European Commission (E.U. Grant Agreement 653477) and the Universidad Complutense de Madrid under the predoctoral grant UCM-Harvard University (CT17/17-CT18/17). We would also like to thank K. Bernlöhr for his helpful technical support.

\section{References}

[1] D. Heck, J. Knapp, J.N. Capdevielle, et al., CORSIKA: a Monte Carlo code to simulate extensive air showers, Forschungszentrum Karlsruhe FZKA 6019 (1998) 1-90.

[2] S. Martínez, F. Arqueros, V. Fonseca, et al., Monte Carlo simulation of the HEGRA cosmic ray detector performance, Nucl. Instrum. Methods Phys. Res., Sect. A 357 (2-3) (1995) 567-579.

[3] K. Bernlöhr, Simulation of imaging atmospheric Cherenkov telescopes with CORSIKA and sim_telarray, Astropart. Phys. 30 (3) (2008) 149-158.

[4] B.S. Acharya, M. Actis, T. Aghajani, et al., Introducing the CTA concept, Astropart. Phys. 43 (2013) 3-18.

[5] D. Morcuende, J. Rosado, J.L. Contreras, et al., Relevance of the fluorescence radiation in VHE gamma-ray observations with the Cherenkov technique, Astropart. Phys. 107 (2019) 26-34.

[6] M. Reininghaus, R. Ulrich, CORSIKA 8 - Towards a modern framework for the simulation of extensive air showers, EPJ Web Conf. 210, 02011 (2019).

[7] D. Morcuende, J.L. Contreras, J. Rosado, et al., A Monte Carlo study of the relevance of fluorescence radiation in VHE gamma-ray observations with Cherenkov telescopes, PoS ( ICRC2017) 839.

[8] M. Tluczykont, D. Hampf, D. Horns, et al., The HiSCORE concept for gamma-ray and cosmic-ray astrophysics beyond $10 \mathrm{TeV}$, Astropart. Phys. 56 (2014) 42-53.

[9] https://github.com/cta-observatory/ctapipe

[10] J. L. Contreras, J. Rosado, F. Arqueros, et al., Feasibility of VHE gamma-ray detection by an array of imaging atmospheric Cherenkov telescopes using the fluorescence technique, PoS ( ICRC2015) 993 